\title{
A Fast, Direct Procedure to Estimate the Desorption Energy for Various Molecular Ices of Astrophysical Interest
}

\author{
Ramón Luna ${ }^{1}$, Ramón Luna-Ferrándiz ${ }^{1}$, Carlos Millán ${ }^{1}$, Manuel Domingo ${ }^{1}$, Guillermo M. Muñoz Caro ${ }^{2}$, \\ Carmina Santonja ${ }^{1}$, and Miguel Á. Satorre ${ }^{1}$ \\ ${ }^{1}$ Centro de Tecnologías Físicas, Universitat Politècnica de València, Plaza Ferrándiz Carbonell, E-03801 Alcoy, Spain; ralunam@ fis.upv.es \\ ${ }^{2}$ Centro de Astrobiología, INTA-CSIC, Carretera de Ajalvir, km 4, Torrejón de Ardoz, E-28850 Madrid, Spain \\ Received 2017 March 3; revised 2017 May 24; accepted 2017 May 25; published 2017 June 14
}

\begin{abstract}
Desorption energy is a relevant parameter when studying the desorption kinetics of an ice under astrophysical conditions. Values reported are generally calculated using at least a desorption experiment and a further data analysis at present. In this work the establishment of a simple rule that relates the desorption energy of a species to the temperature of its desorption peak is explored. The paper presents the results obtained from zeroth-order desorption experiments, based on the use of a quartz crystal microbalance to monitor the loss of weight during desorption of the accreted ice sample under high-vacuum conditions, of nine different molecules covering a wide range of desorption energies. During these experiments, the ice desorption rate reaches a maximum at a certain temperature depending on the molecule. The formula obtained in this study facilitates the estimation of the desorption energy and is valid for all the investigated molecules. Based on these experimental results and simulations, the theoretical expression obtained is valid to calculate desorption energy for zeroth- and first-order desorption experiments under high- or ultrahigh-vacuum conditions using different ice thickness films.
\end{abstract}

Key words: ISM: kinematics and dynamics - ISM: molecules - methods: laboratory: molecular - methods: laboratory: solid state

\section{Introduction}

In many astrophysical scenarios, ices undergo thermal desorption. The study of this process helps us understand the evolution of the physical and chemical conditions in these environments.

To model the thermal desorption of ices, the Polanyi-Wigner equation (Polanyi \& Wigner 1925) is widely used:

$$
-\frac{d N}{d t}=N^{i} A_{i} \exp \left(-\frac{E_{\mathrm{des}}}{R T}\right)
$$

where $N$ is the number of molecules in the solid phase per square centimeter, $i$ is the order of the process, $A_{i}$ is the frequency factor, $E_{\mathrm{des}}$ is the desorption energy, $T$ is the absolute temperature, and $R$ is the constant for ideal gases. Therefore to study a desorption process (to calculate residence time, surface coverage, gas composition, etc.) $E_{\mathrm{des}}$ has to be known, and it is usually determined experimentally.

To obtain this parameter, a long-term project was planned during which the desorption energy for nine different molecules using the same high-vacuum (HV) conditions was established. As a result, a wide range of desorption energies for different substances was established. The procedure used meant carrying out a data analysis of the experimental results collected (summarized in Section 3). Other authors have used the Polanyi-Wigner equation to calculate $E_{\text {des }}$ but have performed alternative experimental procedures. All the methods reported need to perform at least a desorption experiment and a further data analysis of their results. In general the desorption energy values, although coming from different methods, are in reasonable agreement.

Since the desorption energy of the molecules studied in this work covers a wide range of values $\left(4.0-47 \mathrm{~kJ} \mathrm{~mol}^{-1}\right.$ or
$480-5700 \mathrm{~K}$ ) it is possible to find a quantity that allows a direct estimation of the desorption energy.

To carry out this study it is necessary to consider another parameter involved in desorption experiments: the order $i$ of the process. This parameter depends on the kinetics of the desorption and strongly affects the profile of the desorption rate versus temperature. Most desorption processes performed in laboratories under astrophysical conditions undergo a zeroth(related to hundreds of monolayer deposits) or a first-order kinetics (related to a few monolayer deposits) desorption. Values of desorption energy for a specific species do not seem to vary significantly for both orders of kinetics (Luna et al. 2014).

The experiments performed in this laboratory follow a zeroth-order desorption due to the characteristics of the equipment. The desorption rate increases as the temperature increases until all the molecules in the solid state desorb at a certain temperature, which depends on the initial ice mass deposited and its $E_{\text {des. }}$. This is not a maximum in the mathematical sense, since this desorption rate peak is only due to the lack of additional molecules that can desorb. Nevertheless, hereafter this temperature is referred to as a peak, $T_{\text {peak }}$, for reasons of simplicity.

The thickness used for these experiments was more or less the same regardless of the species under study. As a consequence, a similar amount of molecules for all the substances was accreted. Nevertheless, a different $T_{\text {peak }}$ was reached for each species allowing the characterization of each molecular ice by means of this latter parameter.

Since the $T_{\text {peak }}$ is representative of every species desorbed under these experimental conditions, it was necessary to look for a (theoretical, experimental, or both) relationship between this parameter and the $E_{\text {des }}$ to obtain an alternative way to calculate the latter parameter from the former one. This 


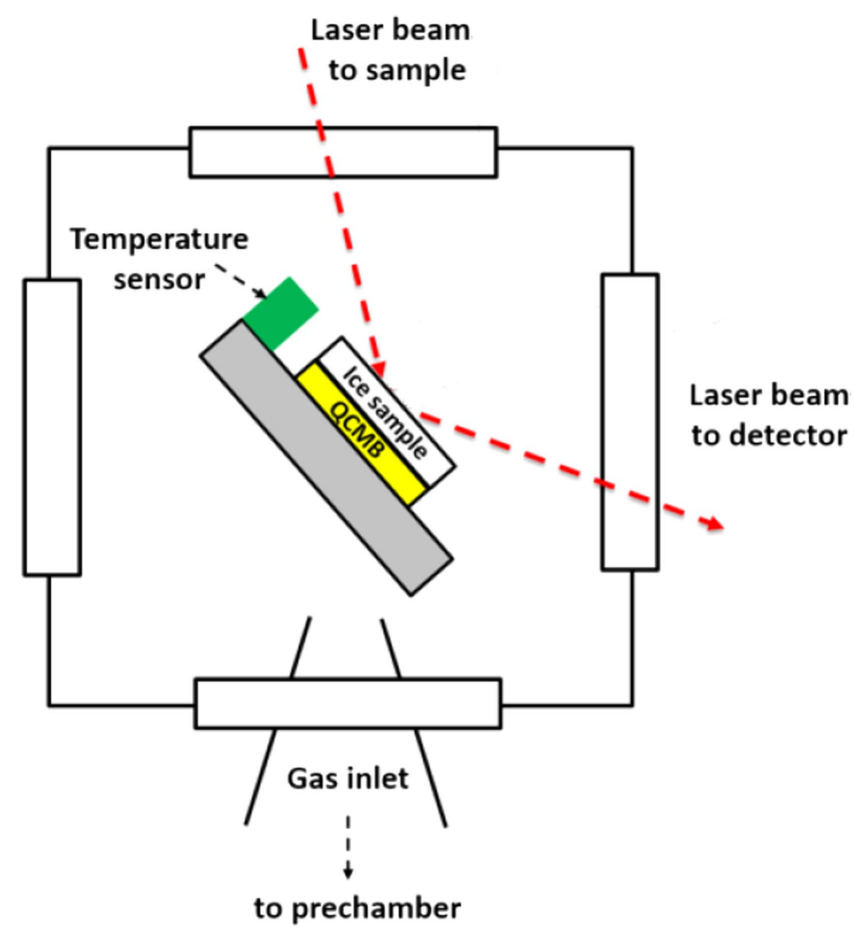

Figure 1. Schematics of the experimental setup.

relationship would provide a fast and simple procedure based on determining the $T_{\text {peak }}$ in a single experiment with no need for further data analysis.

To explain the method developed, Section 2 contains a description of the experimental setup, Section 3 presents the experimental results of the $E_{\mathrm{des}}$ and the $T_{\text {peak }}$ for nine different molecules, in Sections 4 and 5 a theoretical overview of the problem is given, and finally the conclusions are presented in Section 6.

\section{Experimental Setup}

The experiments were carried out in a system working under HV conditions. The main components were a quartz crystal microbalance (QCMB) and a temperature controller (Figure 1).

The pressure of the experiment $\left(10^{-7}\right.$ mbar) was obtained by assembling in the chamber a pair of turbomolecular pumps backed by their corresponding rotary pumps and the help of a closed-cycle helium cryostat acting as a cryopump. The gas under study was previously placed in a prechamber. A constant flow of molecules entered the chamber during the film accretion.

The edge of the cryostat was in thermal contact with the sample holder, where the QCMB - coated with an optically thick, flat plate with a smooth surface-was located (Q-Sense gold coated quartz, $5 \mathrm{MHz}$ ). The temperature of the ice sample (deposited onto the QCMB) was governed by an ITC $503 \mathrm{~S}$ intelligent temperature controller using two silicon diode sensors, with one located just beside the quartz crystal and another beside the heating resistor. This arrangement allowed the temperature to be varied between 13 and $300 \pm 1 \mathrm{~K}$ by means of the resistor. To measure the ice film thickness during the growth, interferometric patterns were obtained using a helium-neon laser $(632.8 \mathrm{~nm})$ system.
Table 1

Values for the $E_{\text {des }}$ Calculated for Nine Different Molecules as Explained in Luna et al. (2012)

\begin{tabular}{lrrr}
\hline \hline \multirow{2}{*}{ Molecule } & \multicolumn{2}{c}{$E_{\text {des }}$} & \\
\cline { 2 - 3 } & \multicolumn{1}{c}{$\mathrm{kJ} \mathrm{mol}^{-1}$} & $\mathrm{~K}$ & $\begin{array}{r}T_{\text {peak }} \\
\pm 1 \mathrm{~K}\end{array}$ \\
\hline Nitrogen & $4.3 \pm 0.2$ & 520 & 25 \\
Carbon monoxide & $6.3 \pm 0.3$ & 760 & 32 \\
Methane & $8.5 \pm 0.4$ & 1020 & 40 \\
Ethylene & $19.3 \pm 1.0$ & 2320 & 70 \\
Ethane & $15.3 \pm 0.8$ & 1840 & 72 \\
Carbon dioxide & $29.3 \pm 1.5$ & 3530 & 95 \\
Ammonia & $31.8 \pm 1.6$ & 3830 & 113 \\
Methanol & $46.2 \pm 2.3$ & 5560 & 163 \\
Water & $47.0 \pm 2.4$ & 5660 & 186 \\
\hline
\end{tabular}

Note. Column 4 represents the $T_{\text {peak }}$ observed for each molecule.

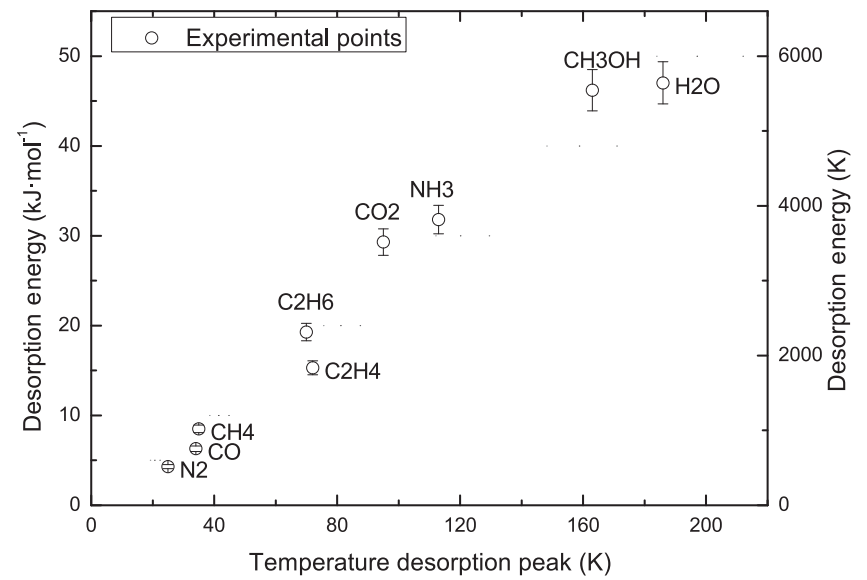

Figure 2. Experimental values of the $E_{\mathrm{des}}$ obtained in the laboratory for nine different molecules plotted vs. their $T_{\text {peak }}$.

\section{Desorption Experiments}

The procedure used to obtain the $E_{\text {des }}$ involves several steps, and this is explained in detail in Luna et al. (2012). In summary, the overall process has four steps: (1) first, contaminants have to be removed from the raw signal; (2) next, the influence of the temperature on the frequency signal of the QCMB is removed; (3) from data obtained in the previous step, the derivative is taken with respect to the temperature to obtain the desorption rate from the frequency signal; and (4) a linear regression of the logarithm of the desorption rate versus $1 / T$ is performed to obtain the $E_{\mathrm{des}}$ from the slope. When using this procedure to calculate the $E_{\mathrm{des}}$ it is not necessary to know the pre-exponential factor.

Table 1 presents the results of the $E_{\text {des }}$ (columns 2 and 3), which was calculated using the procedure described above for all the molecules included in our survey. The desorption experiments were repeated at least five times, and the degree of uncertainty was calculated statistically. Column 4 shows the $T_{\text {peak }}$ experimentally observed for each molecule. These are plotted in Figure 2. This plot shows two facts established in the experiments: the molecules with a higher $E_{\mathrm{des}}$ also display a higher $T_{\text {peak }}$ value, and the $E_{\text {des }}$ seems to follow a linear trend versus the $T_{\text {peak }}$. Therefore, finding a theoretical relationship between these two parameters is imperative. 


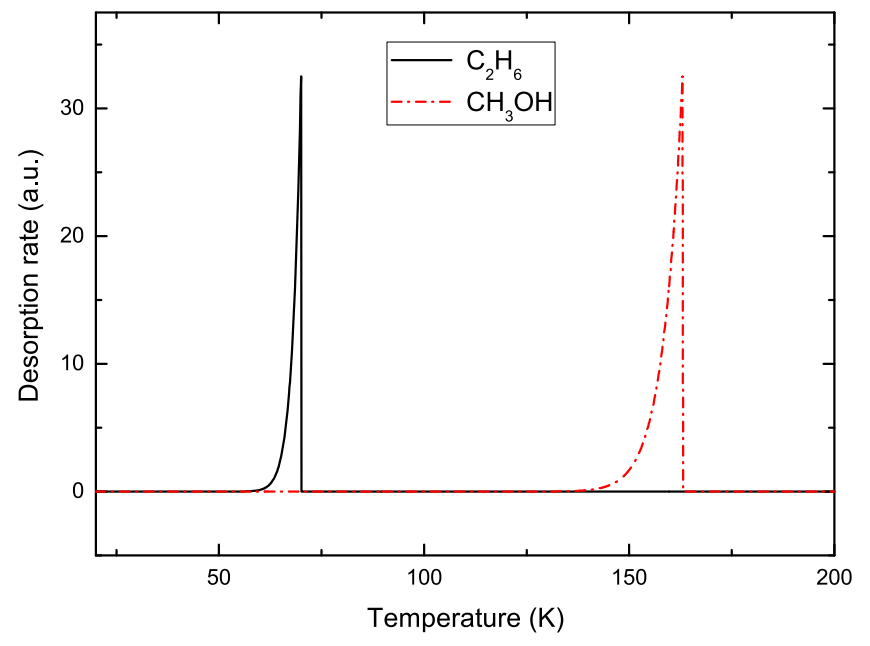

Figure 3. Comparison of the theoretical profile for a zeroth-order desorption process for ethane (solid line) and methanol (dashed line).

\section{Desorption Energy versus Temperature Peak: Zeroth-Order Desorption Survey}

Under the HV conditions of our laboratory, deposits of hundreds of monolayers are prepared in a controlled manner and then their corresponding desorption experiments suffer a zeroth-order process, which implies a multilayer desorption.

A zeroth-order desorption with a constant warming ramp $\beta=\frac{d T}{d t}$ can be modeled from Equation (1) as

$$
-\frac{d N}{d T}=A_{0} \frac{1}{\beta} \exp \left(-\frac{E_{\mathrm{des}}}{R T}\right) .
$$

The exponential factor in Equation (2) indicates that the desorption rate increases as the temperature rises during a typical temperature-programmed desorption experiment. Therefore, the time necessary to desorb all of the initial deposit depends on the initial mass; then the maximum in the desorption peak is always reached when the last molecules desorb from the solid phase. Figure 3 plots the theoretical desorption rate for two different ices undergoing a zeroth-order desorption process: ethane and methanol. For these molecules their $E_{\mathrm{des}}$ differs by approximately $30 \mathrm{~kJ} \mathrm{~mol}^{-1}$ and their $T_{\text {peak }}$ diverges at about $90 \mathrm{~K}$. As can be observed, the higher $E_{\mathrm{des}}$ of methanol is accompanied by higher $T_{\text {peak }}$ values.

To find the theoretical relationship between the $E_{\mathrm{des}}$ and the $T_{\text {peak }}$, a series of steps were performed. First, the number of molecules in the solid phase as a function of temperature was obtained from Equation (2):

$$
-\int_{N_{0}}^{N} d N=\frac{A_{0}}{\beta} \int_{0}^{T} \exp \left(-\frac{E_{\mathrm{des}}}{R T}\right) d T,
$$

obtaining:

$$
N=N_{0}-\frac{A_{0}}{\beta}\left(\frac{E_{\mathrm{des}} \operatorname{ExIn}\left(-\frac{E_{\mathrm{des}}}{R T}\right)}{R}+T \exp \left(-\frac{E_{\mathrm{des}}}{R T}\right)\right),
$$

where ExIn represents

$$
\operatorname{ExIn}(x)=\int_{-\infty}^{x} \frac{\exp (T)}{T} d T
$$

Following this, from Equation (4), using $N_{0}=$ $6.610^{18}$ molecule $\mathrm{cm}^{-2}$ (calculated from the Polanyi-Wigner

\begin{tabular}{|c|c|c|c|c|c|}
\hline \multicolumn{2}{|c|}{$E_{\mathrm{des}}$} & \multicolumn{3}{|c|}{$\beta=1 \mathrm{~K} \mathrm{~min}^{-1}$} & \multirow{2}{*}{$\beta=\underset{0.02 N_{0}}{6 \mathrm{~K} \mathrm{~min}^{-1}}$} \\
\hline $\mathrm{kJ} \mathrm{mol}^{-1}$ & $\mathrm{~K}$ & $N_{0}$ & $10 N_{0}$ & $\begin{array}{c}0.1 N_{0} \\
T_{\text {peak }}(\mathrm{K})\end{array}$ & \\
\hline 5 & 602 & 20.7 & 22.5 & 19.4 & 19.4 \\
\hline 10 & 1203 & 41.5 & 44.8 & 38.6 & 38.6 \\
\hline 20 & 2407 & 81.2 & 87.5 & 75.6 & 75.5 \\
\hline 30 & 3610 & 120.2 & 129.5 & 112.1 & 111.9 \\
\hline 40 & 4813 & 158.8 & 171.0 & 148.2 & 148.0 \\
\hline \multirow[t]{3}{*}{50} & 6017 & 197.1 & 212.1 & 184.0 & 183.7 \\
\hline & & & & $\frac{E_{\mathrm{des}}}{R T_{\text {pak }}}$ & \\
\hline & & 30.9 & 28.8 & 33.1 & 33.1 \\
\hline
\end{tabular}

Table 2

Values of the $T_{\text {peak }}$ Evaluated from Equation (4) Using $N=0$ for Different Values of $\beta$ and the Initial Amount of Molecules

Note. $N_{0}$ is taken as $6.610^{18}$ molecule $\mathrm{cm}^{-2}$.

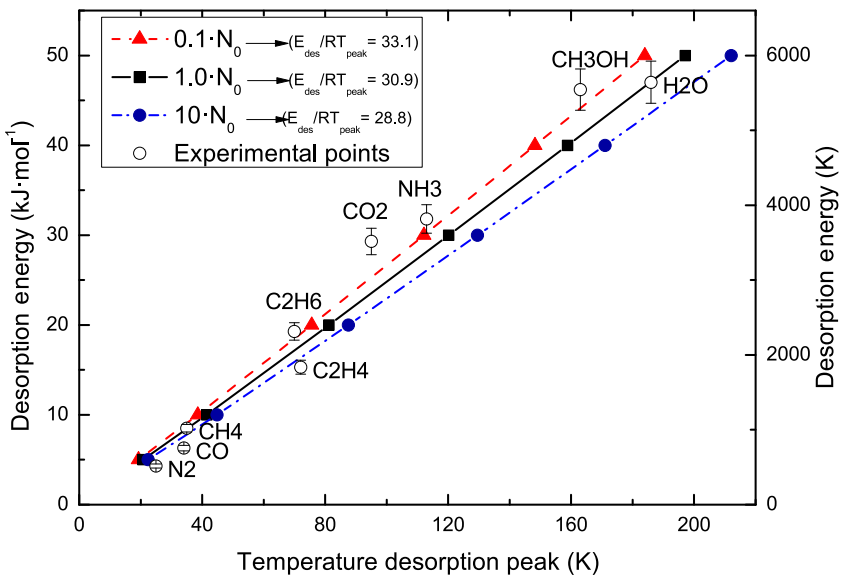

Figure 4. Theoretical values (solid squares) for desorption energy vs. desorption peak for $N_{0}=6.610^{18}$ molecule $\mathrm{cm}^{-2}, \beta=1 \mathrm{~K} \mathrm{~min}^{-1}\left(1 / 60 \mathrm{~K} \mathrm{~s}^{-1}\right)$, and $A_{0}=10^{30}$ molecule s $\mathrm{sm}^{-1}$. Experimental results were obtained in the laboratory for different molecules (open circles). Solid circles and triangles represent how this relationship would change if the initial mass deposited were $10 \times N_{0}$ or $0.1 \times N_{0}$, respectively.

equation using the experimental values of the $E_{\mathrm{des}}$ and the $\left.T_{\text {peak }}\right)$ and setting the final amount of molecules in the solid state, $N=0$, an implicit relationship between the $E_{\text {des }}$ and the $T_{\text {peak }}$ is reached. As a consequence, it was possible to evaluated this theoretical function for six different values of $E_{\text {des }}$ in the range of $5-50 \mathrm{~kJ} \mathrm{~mol}^{-1}(602-6017 \mathrm{~K})$ since most of the abundant astrophysical molecules are included in this interval. The parameters used were $\beta=1 \mathrm{~K} \mathrm{~min}^{-1}\left(1 / 60 \mathrm{~K} \mathrm{~s}^{-1}\right.$, the temperature ramp used in the desorption experiments) and $A_{0}=10^{30}$ molecule $\mathrm{s}^{-1} \mathrm{~cm}^{-2}$ (taken as representative of the frequency factor for molecules undergoing a zeroth-order desorption process; Luna et al. 2015). The results are presented in columns 1 and 2 of Table 2 and are also plotted in Figure 4, where the $E_{\text {des }}$ is represented against the $T_{\text {peak }}$ (solid squares). As can be seen, the results can be fitted to a straight line (solid line in Figure 4). This plot starts at $20 \mathrm{~K}$ since no lower experimental values of the $T_{\text {peak }}$ were reached. The slope of the linear fit delivers the value of the ratio that was sought. In this case, the quotient obtained was

$$
\frac{E_{\mathrm{des}}}{R T_{\text {peak }}}=30.9 \text {, }
$$


where the units of the $E_{\mathrm{des}}$ are $\mathrm{Jmol}^{-1}, T_{\text {peak }}$ in $K$, and the value of $R$ is $8.314 \mathrm{~J} \mathrm{~mol}^{-1} \mathrm{~K}^{-1}$.

To compare this theoretical expression with the experimental values obtained in this work, in Figure 4 the experimental values for the $E_{\text {des }}$ versus the $T_{\text {peak }}$ of the nine molecules listed in Table 1 are also plotted (open circles). As can be observed, the experimental results fit reasonably well to the theoretical line obtained (solid line). Deviations from the straight line may be for different reasons: (1) each molecule has a different density and thus the initial amount deposited is different since the method used in this work is based on depositing approximately the same thickness for all the experiments; (2) different molecules have different values for their frequency factor, which has been assumed as a constant; (3) the frequency factor is also affected by temperature, as noted in Luna et al. (2015); and (4) in addition, other experimental errors. However, although there are many possible sources of errors the previous relationship agrees reasonably well with the experimental results.

Once this expression was presented, it was also necessary to analyze how experimental data from other laboratories can fit this expression. As a first step the initial amount deposited by different authors in their experiments was analyzed. The thickness deposited in each laboratory depends on that laboratory's instrumental constraints and needs, so it is necessary to study how thickness affects the $T_{\text {peak }}$ variation. For this reason the previous procedure was repeated for two different cases: if the initial mass was taken as $10 N_{0}$ or if it was taken as $0.1 N_{0}$. The results are presented in columns 4 and 5 of Table 2 and are also represented in Figure 4, with a dashed dotted line $\left(10 N_{0}\right)$ and a dashed line $\left(0.1 N_{0}\right)$. For these two cases, despite the fact that the deposited mass is increased or decreased by a factor of 10 , the ratio $\frac{E_{\text {des }}}{R T_{\text {peak }}}$ varies by at most around $10 \%$ (bottom row in Table 2). This latter variation is of the order of the deviation observed when the $E_{\text {des }}$ for the same molecules is obtained by different authors. This result can be interpreted as follows: under our experimental conditions, similar results can be obtained for the $E_{\text {des }}$ using deposits within the range of $0.2-20 \mu \mathrm{m}$ (corresponding to two different orders of magnitude), which covers many laboratory requirements.

Concerning the parameter $\beta$, some laboratories working under ultrahigh-vacuum (UHV) conditions usually use $\beta=6 \mathrm{~K} \mathrm{~min}^{-1}\left(0.1 \mathrm{~K} \mathrm{~s}^{-1}\right)$. Under these conditions, two opposing effects have to be taken into account. For those experiments the thickness deposited is around two orders of magnitude lower and as a result a lower $T_{\text {peak }}$ would be expected, but as the $\beta$ is higher than the value used for the experiments carried out in this work, this fact would provide a higher $T_{\text {peak }}$; therefore there is a compensating effect. To theoretically study this effect, a deposit of $0.02 N_{0}$ and $\beta=6 \mathrm{~K} \mathrm{~min}^{-1}$ was used. The results obtained are presented in column 6 of Table 2 and are not plotted in Figure 4 as they exactly match the results previously obtained for $0.1 N_{0}$. As can be seen, these results are on the edge of the area defined by the two straight lines, covering the dispersion of $10 \%$ previously obtained. Therefore, for higher values of $\beta$, under UHV conditions, the results will be within this mentioned area.

In conclusion, for a zeroth-order process, it is possible to experimentally estimate the $E_{\mathrm{des}}$ from a single desorption experiment using the relationship obtained in this work. No significant variations are obtained using very different experimental conditions, covering a broad range of initial deposited mass and warming rate values.

\section{Study Extended to a First-Order Desorption Process}

For a first-order desorption experiment, the concept of desorption peak is inherent to its mathematical expression. In this case, Equation (1) becomes

$$
-\frac{d N}{d T}=N \frac{A_{1}}{\beta} \exp \left(-\frac{E_{\mathrm{des}}}{R T}\right) .
$$

This expression leads to a desorption rate profile where a maximum is reached since it represents a product of the decreasing amount of molecules in the solid phase (dashed line in Figure 5, left panel) and an exponential factor increasing with temperature (dotted line in Figure 5, left panel).

For these first-order processes, the position of the maximum achieved does not depend on the initial mass of the ice deposited. Figure 5 (right panel) shows the theoretical desorption rate for methanol for several different initial masses $\left(1.00 N_{0}, 0.90 N_{0}, 0.75 N_{0}\right.$, and $\left.0.50 N_{0}\right)$. The peak position remains constant for any of the initial masses, as can be observed.

An analytical expression can be obtained to calculate the position of this maximum if from Equation (7) the derivative with respect to the temperature is taken.

$$
\begin{aligned}
\frac{d^{2} N}{d T^{2}}= & -\frac{d N}{d T} \frac{A_{1}}{\beta} \exp \left(-\frac{E_{\mathrm{des}}}{R T}\right) \\
& -N \frac{A_{1}}{\beta}\left(\frac{E_{\mathrm{des}}}{R T^{2}}\right) \cdot \exp \left(-\frac{E_{\mathrm{des}}}{R T}\right)
\end{aligned}
$$

using Equations (7) and (8) and equating to zero leads to

$$
\frac{E_{\mathrm{des}}}{R T_{\text {peak }}^{2}}=\frac{A_{1}}{\beta} \exp \left(-\frac{E_{\mathrm{des}}}{R T_{\text {peak }}}\right),
$$

where $T_{\text {peak }}$ is the temperature at which the maximum desorption is reached. As can be seen from this expression, for a first-order desorption process, the $T_{\text {peak }}$ does not depend on the initial amount of molecules in the deposit.

An estimation for the relationship between the $E_{\mathrm{des}}$ and the $T_{\text {peak }}$ (Attard \& Barnes 1998) was based on experiments under conditions of high temperature related to catalysis that are very different than those related to astrophysical scenarios. For these conditions, the authors proposed an empirical rule of thumb, $E_{\text {des }}=\frac{T_{\text {peak }}}{4}$, where the units of $E_{\text {des }}$ are $\mathrm{kJ} \mathrm{mol}^{-1}$ and $T_{\text {peak }}$ in $K$. Reordering this expression to be similar to Equation (6) changes it to

$$
\frac{E_{\mathrm{des}}}{R T_{\text {peak }}}=30.1 \text {. }
$$

Alternatively, the problem has been addressed analytically for the first time in this work, applying conditions related to astrophysical environments. The following expression was used to obtain a representative value of the quotient:

$$
\frac{E_{\mathrm{des}}}{R T_{\text {peak }}}=\operatorname{ProductLog}\left(\frac{A_{1} T_{\text {peak }}}{\beta}\right) \text {, }
$$

where ProductLog is the Lambert function (Corless et al. 1996). To obtain a result representative of this function, the following values for the parameters involved were used: 

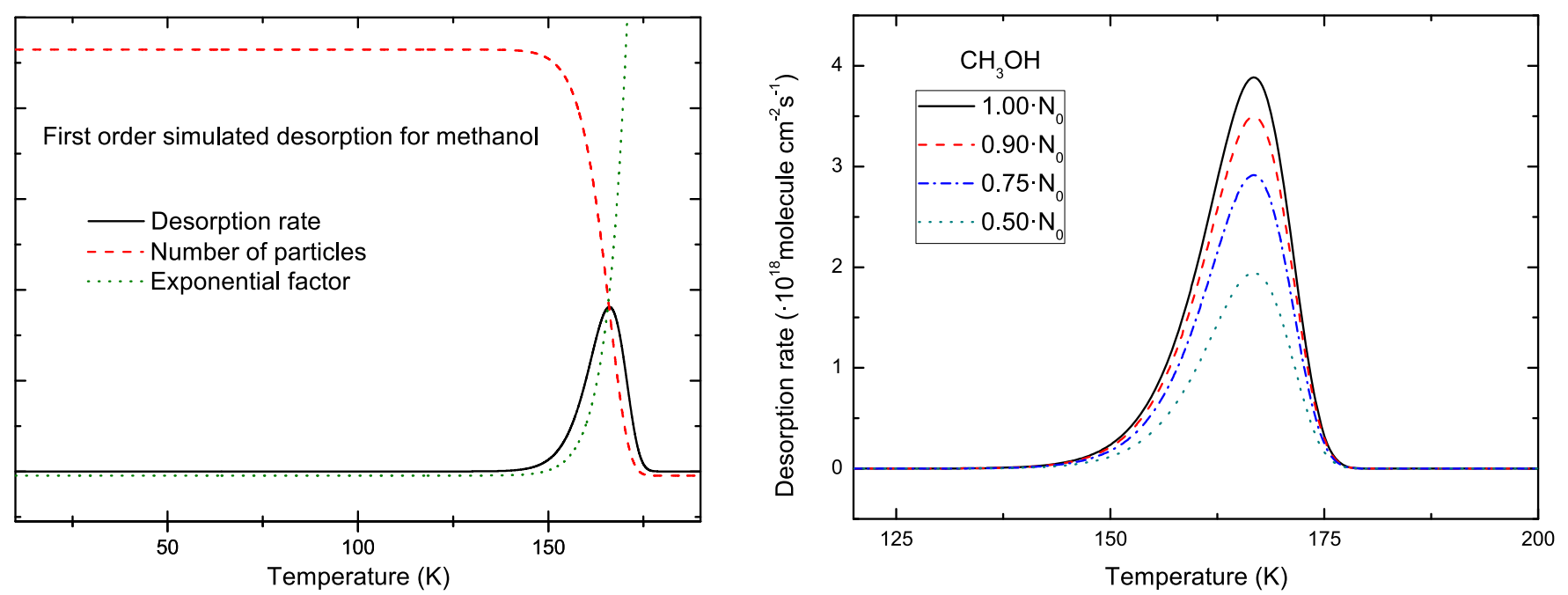

Figure 5. Left panel: The desorption rate (solid line) in a theoretical first-order desorption process. The dashed line represents the amount of molecules in the solid state. The dotted line represents how the exponential factor of the Polanyi-Wigner equation varies for this process. Right panel: The desorption rate for methanol at four different initial masses for a theoretical first-order desorption process. The desorption peak is not influenced by the initial amount of ice.

$A_{1}=10^{12} \mathrm{~s}^{-1}$, as it is commonly assumed for first-order desorption processes; $\beta=6 \mathrm{~K} \mathrm{~min}^{-1}$, since most laboratories performing first-order desorption processes use this heating rate; and $25-170 \mathrm{~K}$, used for the temperature range, in which most abundant astrophysical ices desorb. Using these values, the Lambert function was calculated for all the temperatures (see Figure 6) and the mean value was assessed, obtaining

$$
\frac{E_{\text {des }}}{R T_{\text {peak }}}=31.0 \pm 0.6
$$

This value is very similar to the previous one obtained for zeroth-order desorption processes.

To validate the procedure established in this work, the ratio $\frac{E_{\text {des }}}{R T_{\text {paak }}}=31.0$ has been compared to several values obtained from the data reported in the literature for different molecules.

In Table 3, some values for desorption energy are shown for different molecules and their corresponding desorption peak; they were collected from the literature from experiments reported as being performed under astrophysical conditions and following a first-order desorption or showing a profile characteristic of this type of desorption process. Column 5 shows the ratio $E_{\text {des }} /\left(R T_{\text {peak }}\right)$. As can be observed, the experimental ratio is very close to the one theoretically obtained.

The result obtained in this work is very similar to that proposed as a rule of thumb by Attard \& Barnes (1998), although a very different temperature interval was used. This simple expression that allows a fast estimation of $E_{\mathrm{des}}$ was later used by various authors and has been published in astrophysical journals (Öberg et al. 2005; Martín-Doménech et al. 2014). For this reason, it is important to confirm this proposed relationship both theoretically and experimentally and apply it to the common astrophysical ice molecules and to a large interval of temperatures concerning the most abundant astrophysical ices.

In addition, both the theoretical results obtained for firstorder desorption and those of Attard \& Barnes (1998) are very similar to the ones obtained here for zeroth-order desorption processes. Therefore, this experimental relationship seems to hold true under a wide variety of experimental conditions

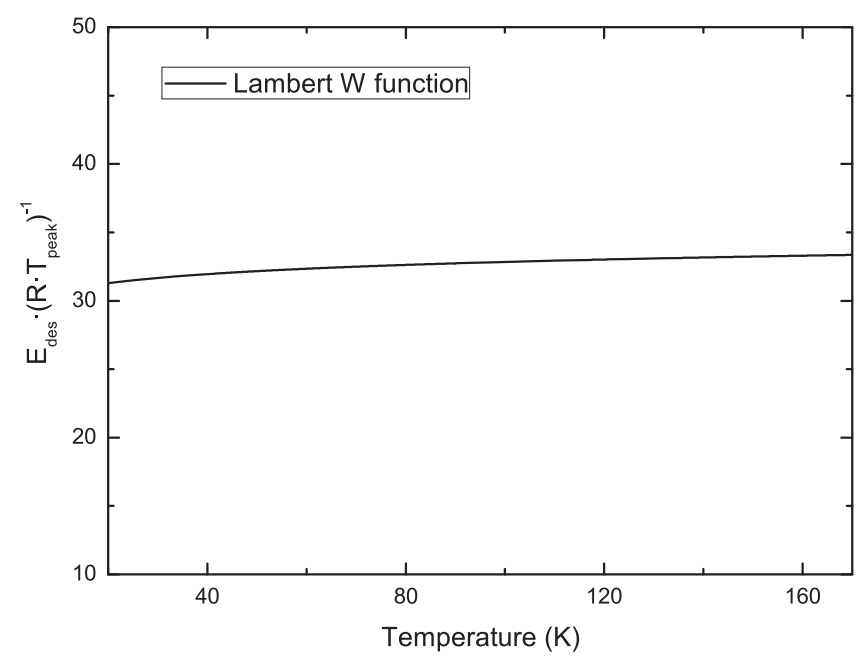

Figure 6. Plot of the Lambert function for $\frac{A_{1} T_{\text {peak }}}{\beta}$ in the interval $25-170 \mathrm{~K}$.

Table 3

Values for Desorption Energy and Desorption Peak Temperature Reported in the Literature for First-order Desorption Processes

\begin{tabular}{|c|c|c|c|c|}
\hline \multirow[t]{2}{*}{ Molecule } & \multicolumn{2}{|c|}{$E_{\mathrm{des}}$} & \multirow{2}{*}{$\begin{array}{c}T_{\text {peak }} \\
\mathrm{K}\end{array}$} & \multirow{2}{*}{$\begin{array}{c}\text { Ratio } \\
\frac{E_{\text {des }}}{R T_{\text {peak }}}\end{array}$} \\
\hline & $\mathrm{kJ} \mathrm{mol}^{-1}$ & K & & \\
\hline Nitrogen (a) & 6.65 & 800 & 26 & 30.7 \\
\hline Ammonia (b) & 24.3 & 2920 & 94 & 31.1 \\
\hline Water (c) & 39.9 & 4800 & 140 & 34.3 \\
\hline Methanol (d) & 41.5 & 4990 & 146 & 34.2 \\
\hline
\end{tabular}

References. (a) Bisschop et al. (2006), (b) Bolina \& Brown (2005), (c) Brown \& Bolina (2007), (d) Doronin et al. (2015).

within an acceptable degree of uncertainty and can be applied to initially evaluate the $E_{\text {des }}$ from a single desorption experiment for both desorption kinetics (zeroth and first order).

\section{Conclusions}

When these results are applied to astrophysical scenarios, the influence of water must be taken into account since some ice 
mantle polar components may be mixed with water (Boogert et al. 2015). However, the study of mixtures where water is the main component has been addressed by several authors and typically all these molecules behave in a similar way, presenting two main desorption peaks: a first peak corresponding to its own desorption energy and an additional peak when this molecule desorbs simultaneously with water (Collings et al. 2004; Martín-Doménech et al. 2014). This retaining behavior is not exclusive of a polar molecule like water but is also observed for other nonpolar molecules, e.g., carbon dioxide (Satorre et al. 2009).

Calculations of desorption energy are necessary to interpret desorption processes occurring in many astrophysical scenarios. Methods used so far have involved an elaborate procedure including both experiments and data analysis. In this work, the possibility of reducing the process necessary to asses this parameter has been addressed. The goal of the study has been to find a relationship between the $E_{\text {des }}$ and the $T_{\text {peak }}$.

Under the experimental conditions used in this work, it was found that as the $E_{\mathrm{des}}$ increases for different molecules, the $T_{\text {peak }}$ also increases. The data follow an almost linear trend.

A theoretical relationship of $\frac{E_{\mathrm{des}}}{R T_{\text {peak }}}=30.9$ has been calculated under our experimental conditions. The experimental values obtained in our laboratory for zeroth desorption processes fit well to the theoretical expression obtained. To extend this result to other laboratories, research was conducted on how different initial ice masses may affect this relationship. As a result, it was established that for initial masses in the range from 0.1 to $10 N_{0}\left(N_{0}\right.$ being the initial amount of molecules deposited) the deviation obtained for $E_{\mathrm{des}}$ is similar to that achieved according to previous research.
In addition, when UHV conditions are addressed, two factors (thickness and $\beta$ ) with opposing influences mean that the relationship is also valid. Even for the case of first-order desorption processes a very similar relationship is obtained.

Therefore, using $\frac{E_{\text {des }}}{R T_{\text {peak }}}=30.9$ provides a very quick method of estimating the $E_{\mathrm{des}}$ for zeroth- and first-order desorption processes covering a broad range of initial ice mass and warming-up rate values.

This work was supported by the Plan Nacional FIS201348087-C2-2-P, AYA2014-60585-P, and AYA2015-71975REDT of the Ministerio de Economía y Competitividad.

\section{References}

Attard, G., \& Barnes, C. 1998, Surfaces (Oxford: Oxford Univ. Press) Bisschop, S. E., Fraser, H. J., Öberg, K. I., et al. 2006, A\&A, 449, 1297 Bolina, A. S., \& Brown, W. A. 2005, SurSc, 598, 45

Boogert, A. C. A., Gerakines, P. A., \& Whittet, D. C. B. 2015, ARA\&A, 53,541

Brown, W. A., \& Bolina, A. S. 2007, MNRAS, 374, 1006

Collings, M. P., Anderson, M. A., Chen, R., \& Dever, J. W. 2004, MNRAS, 354,1133

Corless, R. M., Gonnet, G. H., Hare, D. E. G., et al. 1996, Adv. Comput. Math, 5,329

Doronin, M., Bertin, M., Michaut, X., et al. 2015, JChPh, 143, 084703

Luna, R., Millán, C., Domingo, M., et al. 2012, Vacuu, 86, 1969

Luna, R., Millán, C., Domingo, M., et al. 2015, Vacuu, 122, 154

Luna, R., Satorre, M. Á., Santonja, C., \& Domingo, M. 2014, A\&A, 566, A27

Martín-Doménech, R., Muñoz Caro, G. M., Bueno, J., \& Goesmann, F. 2014, A\&A, 564, A8

Öberg, K. I., van Broekhuizen, F., Fraser, H. J., et al. 2005, ApJL, 621, L33

Polanyi, M., \& Wigner, E. 1925, ZhPhy, 33, 429

Satorre, M. Á., Luna, R., Millán, C., et al. 2009, P\&SS, 57, 250 